\title{
New Methods for the Fabrication of Composites for Supercapacitor Electrodes with High Active Mass Loading
}

\author{
I. Zhitomirsky \\ Department of Materials Science and Engineering, McMaster University \\ Hamilton, Ontario, Canada
}

Electrochemical supercapacitors are being developed for novel applications in electric and hybrid vehicles, buses, aircrafts and various electronic devices. The success of the supercapacitor technology will depend largely on the ability to achieve good electrochemical performance of advanced electrode materials at practically important high active mass loadings and high charge-discharge rates. The purpose of this study is the development of advanced composite metal oxide-carbon nanotube and metal oxide-graphene electrodes for electrochemical supercapacitors with high active mass loading, high areal and gravimetric capacitances, good cyclic stability and low impedance. The approach is based on the development of new dispersing and capping agents for synthesis and colloidal processing of oxide nanoparticles, new dispersing agents for carbon nanotube and graphene and new techniques for composite design. Chelating organic molecules with strong polydentate bonding to metal oxide surface were used as capping and dispersing agents with superior control of nanoparticle size and dispersion. Small aromatic molecules and materials from bile acid family allowed excellent dispersion of carbon nanotubes and graphene. New chelating polymers and chelating polymer complexes with redox properties allowed superior codispersion of electrode components and were utilized as advanced redox-active binders for electrodes. Liquid-liquid extraction techniques, extraction strategies and extractors were developed for agglomerate free nanoparticle processing. Electrostatic heterocoagulation techniques were developed for the design of advanced electrodes. Advanced supercapacitor electrodes were developed with areal capacitance of $8 \mathrm{~F} \mathrm{~cm}^{-2}$, capacitance retention at high charge-discharge rate above $50 \%$, low impedance, good cyclic stability and high power-energy characteristics. 